\title{
Uterine Artery Embolization for Symptomatic Leiomyomata
}

\author{
Hans van Overhagen $\cdot$ Jim A. Reekers
}

Received: 3 June 2014/ Accepted: 10 September 2014/Published online: 4 December 2014

(C) Springer Science+Business Media New York and the Cardiovascular and Interventional Radiological Society of Europe (CIRSE) 2014

\section{Introduction}

Transcatheter uterine artery embolization (UAE) for treatment of fibroids was first reported by Ravina in 1995 [1]. The continuous advance in the field of vascular Interventional Radiology has facilitated UAE, through the development of low profile catheters and particulate emboli such as polyvinyl alcohol particles (PVA), PVA microspheres and trisacryl gelatine microspheres (TAGM). Prospective randomized studies such as the EMMY and REST trial that compare UAE with hysterectomy have proven scientifically that there is a place for UAE in the treatment of women with symptomatic fibroids [2, 3]. The QOL 5 years after hysterectomy or embolization is equal. Several registries and non-randomized trials have supported these findings. The trials have shown that UAE should be offered to women with symptomatic fibroids causing menorrhagia, as an alternative to hysterectomy (Level 1). The present document provides quality assurance guidelines regarding UAE for the treatment of symptomatic uterine fibroids.

\section{Definitions}

Technical success is an occlusion or marked reduction of blood flow in both uterine arteries. Successful embolization

H. van Overhagen $(\bowtie)$

Department of Radiology, Hagaziekenhuis, Leyweg 275, 2545 CH The Hague, The Netherlands e-mail: h.voverhagen@ hagaziekenhuis.nl

\section{J. A. Reekers}

Department of Radiology, Academic Medical Centre, Meibergdreef 9, 1105 AZ Amsterdam, The Netherlands e-mail: j.a.reekers@amc.uva.nl of only one uterine artery is considered a technical failure unless only a single uterine artery is present.

Clinical success is the resolution or satisfactory improvement of the patients presenting symptoms, such as menorrhagia or bulk-related pain, bloating, urinary urge, or constipation, without additional therapy.

Non-target embolization is the unintended release of an embolic agent into a vascular territory outside the targeted area. In the pelvis, the areas of concern are the ovaries, urinary bladder, intestine, muscles, and nerves, in which non-target embolization can result in symptoms of pain and/or infarction, the possibility of temporary or permanent disability and premature menopause.

Post-embolization syndrome is the occurrence of pelvic pain, low-grade fever, nausea, vomiting, loss of appetite and malaise in the first few days after UAE.

Endometritis is defined as inflammation of the inner lining of the uterus (endometrium) after UAE, which presents as pelvic pain, watery vaginal discharge, fever and/or leukocytosis, and can occur days to weeks after the procedure. It may be due to infectious and non-infectious causes.

Leiomyoma or Fibroid infection is a bacterial infection of one or more fibroids as a result of (i) colonization of devitalised fibroid tissue by blood-borne pathogens or (ii) the ascent of vaginal organisms. Symptoms and signs include abdominal or pelvic pain, fever and/or leukocytosis.

Uterine (myometrial) infection is defined as infection of the uterus, possibly as a result of necrosis of all or part of the uterus, which manifests as abdominal or pelvic pain, vaginal discharge, fever and/or leukocytosis.

Transcervical leiomyoma or fibroid expulsion is defined as the detachment of leiomyoma tissue from the uterine wall and subsequent transvaginal passage, most commonly 
occurring with submucosal fibroids that have narrow points of attachment. This process may be associated with uterine contractions, abdominal pain, fever, nausea, vomiting and vaginal bleeding or discharge.

Levels of evidence according to the Oxford Centre for Evidence-based Medicine are as follows: Level 1A systematic review (SR) of RCTs. 1B individual RCT. 2A SR of cohort studies. 2B individual cohort study. 3A SR of case-control studies. 3B individual case-control study. 4 case series. 5 expert opinions.

\section{Pre-treatment Imaging}

Cross-sectional imaging (Fig. 1) by ultrasound or Magnetic Resonance Imaging (MRI) is performed to confirm the diagnosis of leiomyoma and to rule out other pathology. In assessing suitability fsor UAE, MRI with iv Gadolinium enhancement is the preferred imaging technique (Level 2). MRI provides considerable additional information compared to transabdominal ultrasonography with regard to the number and location of fibroids [4]. MRI has also been shown to be superior to transvaginal ultrasonography in assessing correct positioning and uterine wall embedment of the fibroids [5]. In addition, MRI criteria used for the selection of suitable UAE patients are reported to show good inter- and intra-observer reproducibility [6]. Findings on MRI that are considered important in determining the suitability for UAE treatment are the size of the uterus and the fibroids, the number and location of the fibroids, the presence of pedunculated fibroids, signal intensity of fibroids on T1and T2-weighted images, viability of the fibroids, the presence or absence of adenomyosis and coincidental pathologies that may change patient management [6-8].

MRI is usually performed by obtaining coronal, sagittal and axial T2- and T1-weighted sequences. Gadolinium should be administered to determine the viability of the individual fibroids. Fibroids that show infarction prior to embolization are less likely to shrink after treatment. 3D contrast-enhanced MRA has been shown to be useful for mapping the pelvic vasculature including the ovarian arteries and to identify the optimal tube angle to facilitate catheterization of the uterine arteries during embolization [7].

\section{Indications for Treatment and Contraindications}

UAE is indicated for uterine fibroids causing significant lifestyle-altering symptoms, specifically heavy menstrual

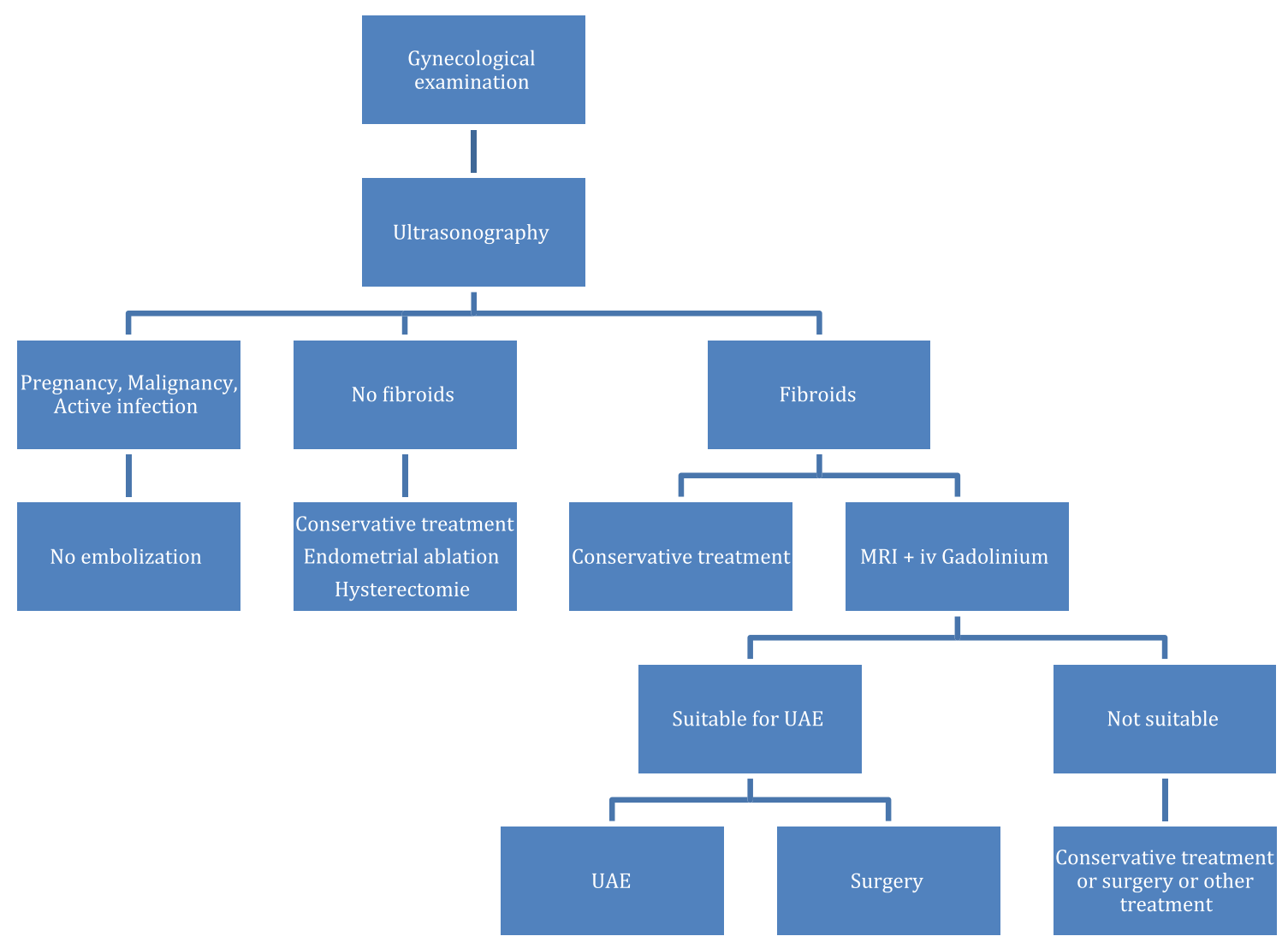

Fig. 1 Flow chart in heavy menstrual bleeding 
bleeding, severe dysmenorrhoea or anaemia (Level 1). UAE is also indicated in fibroids that cause pain or a mass effect on the bladder or intestines (Level 3).

Contraindications include a viable pregnancy, active infection of the uterus and malignancy of the uterus or the ovaries unless the procedure is being performed for palliation or as an adjunct to surgery (Table 1).

Relative contraindications for UAE specifically include the desire to maintain childbearing potential because preservation of fertility is not assured in the current literature (Table 2). However, uncomplicated pregnancies and normal deliveries have been reported after $\operatorname{UAE}[9,10]$. Thus, this procedure may be considered for women who are not candidates for myomectomy, however, not as a first choice option (Table 1).

Traditionally, UAE has been used to treat fibroids with relatively small diameters after anecdotal reports of limited results and increased complications in larger $(>8 \mathrm{~cm})$ fibroids. However, two recent studies showed no different clinical results or complication rates after UAE of large $(>10 \mathrm{~cm}$ ) fibroids in a total of 100 patients [11, 12] (Level $3)$.

The presence of pedunculated subserosal fibroids, defined as a uterine fibroid tumour, with a stalk diameter at least $50 \%$ narrower than the diameter of the tumour is considered a relative contraindication because this might cause torsion of the stalk, ischaemic necrosis of the tumour or separation of the tumour from the uterus [13]. There are a few cases in the literature reporting complications due to septic pedunculated subserosal leiomyoma requiring

\begin{tabular}{l} 
Table 1 Uterine fibroid embolization indications and \\
contraindications \\
\hline Indications \\
Heavy menstrual bleeding \\
Mechanical complaints, such as pain, pressure, dyspareunia, \\
urinary urgency and frequency \\
Contradictions absolute \\
Pregnancy \\
Malignancy of uterus of ovaries (unless palliation or adjunct to \\
surgery) \\
Active uterine infection \\
Contradictions relative \\
Pregnancy wish in the future \\
Certain anatomical features \\
Pedunculated subserosal fibroids with thin stalks \\
Common arterial supply of uterus and ovaries \\
Presence of IUD \\
General contraindications for endovascular procedures \\
Contrast material allergy \\
Impaired renal function \\
Coagulopathy \\
\hline
\end{tabular}

Table 2 Uterine artery embolization outcome

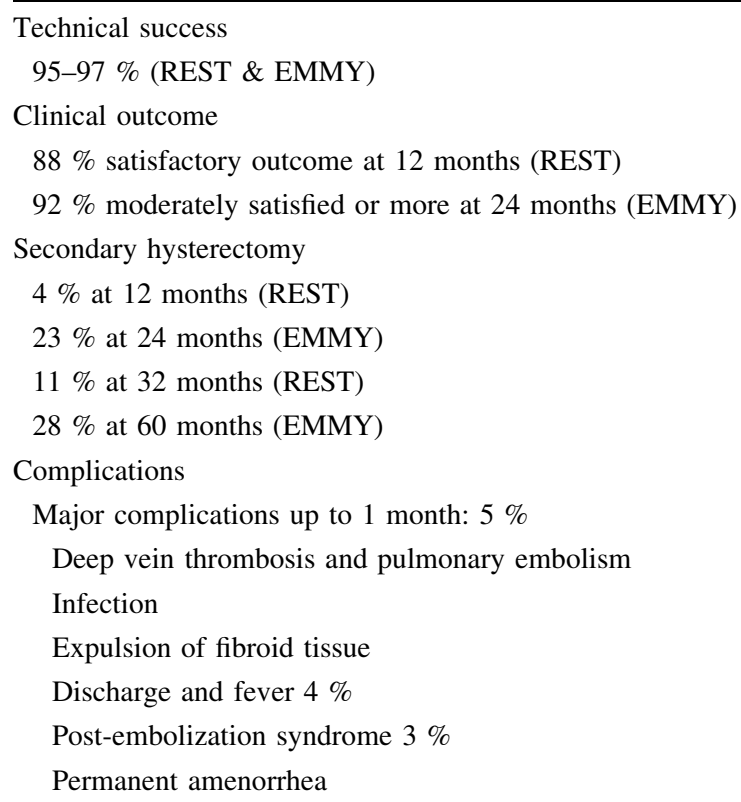

surgery, but the exact incidence of complications after embolization of such lesions is unknown. There are a few reports in the literature that support the use of UAE for pedunculated subserosal fibroids but these are limited [14]. Thus, at present, the embolization of pedunculated (tumour stalk at least $50 \%$ narrower than the tumour itself) subserosal uterine fibroids remains controversial (Level 3).

Another contraindication is the presence of a common arterial supply of the uterus and (one or both of) the ovaries, in those cases when the uterus cannot be embolized selectively. Embolization of the ovaries can lead to premature menopause especially in women older than 45 years of age (Level 5).

The presence of an intrauterine device (IUD) has traditionally been considered a contraindication for uterine embolization, but a recent series of 20 women, accidentally embolized with an IUD in situ did not show any infectious side effects [15] (Level 4).

Relative contraindications to any endovascular intervention include coagulopathy, contrast material allergy and impaired renal function, which can be treated preembolization.

\section{Patient Preparation}

All possible candidates for UAE should undergo assessment by a gynaecologist with training and experience in gynaecologic care. The examination should confirm the diagnosis of leiomyomata and exclude a viable pregnancy and/or active infection or malignancy of the vagina, cervix, uterus and ovaries [16]. 
All candidates should also undergo assessment by the attending interventional radiologist in order to be informed about the procedure, clinical success rates, complications and follow-up.

A recent complete blood count should be available for patients with heavy menstrual bleeding. For patients with a history suggestive of an underlying bleeding disorder, further tests such as an activated partial thromboplastin time and prothrombin time with international normalized ratio should be acquired [16].

Renal function should be acquired from high-risk patients. In cases of impaired renal function (eGFR $<60 \mathrm{ml} /$ $\min / 1.73 \mathrm{~m}^{2}$ ), patients should be treated according to the European Society of Urogenital Radiology international guidelines [17].

It is believed that the use of gonadotropin-releasing hormone $(\mathrm{GnRH})$ agonists by patients should be discontinued since it may hinder the technical success of UAE. However, in a recent study, the use of GnRH agonists before embolization of large fibroids was safe and did not prevent technically successful UAE [18] (Level 4).

It may be prudent to have an IUD removed prior to the procedure in order to avoid infection (see indications and contraindications section above).

\section{Equipment Specifications}

In order to perform UAE, the use of a dedicated DSA C-ARM unit adequately equipped with a large matrix providing high-quality imaging and sufficient magnification is indispensable. Radiation exposure and radiation risk have to be considered, especially because the patients are often (in their last period) of childbearing age and radiation-sensitive organs such as the ovaries lie in the radiation beam [19]. The estimated absorbed ovarian dose during $\mathrm{UAE}$ is greater than that during common fluoroscopic procedures, but the dose is unlikely to result in acute or long-term radiation injury to the patient or increase the genetic risk to the patient's future children [20]. It should be noted that pulsed fluoroscopy, greater filtration and optimal technical use of the system during UAE reduce the radiation exposure [21, 22]. Fluoroscopy time and the use of oblique or magnified fluoroscopy should be limited. The contribution of radiation dose from angiographic runs is reported to be much less significant [23].

The procedure must be performed in a well-organized hospital. The unit should have access to urgent gynaecological, anaesthetic and vascular surgical support if required.

The CIRSE or equivalent checklist should be used and followed for patient safety. A variety of dedicated embolization catheters and embolic materials is essential for the safety and effectiveness of the embolization procedures.
Standard Materials Include

- $5 \mathrm{~F}$ sheaths.

- 4-5 Fr catheters such as Cobra, Roberts uterine and others.

- Microcatheters.

- $0.035^{\prime}, 0.018^{\prime}$ and $0.014^{\prime}$ guide-wires.

- Embolic materials such as particles $(350-900 \mu)$, microspheres $(500-900 \mu)$ or gelfoam.

- Percutaneous closure devices are optional.

Colour Doppler ultrasound for US-guided puncture is optional

\section{Procedure}

Arterial access is usually obtained through the common femoral artery. Whereas some authors prefer bilateral access for embolization of both uterine arteries, others prefer unilateral approach with sequential embolization of the uterine arteries which is feasible using a Waldman loop or reversedtype catheters in the majority of cases. Theoretically, a unilateral approach has the advantage of only one arterial puncture but the disadvantage of longer fluoroscopy time [24]. The internal iliac artery is usually catheterized more easily with a contralateral approach over the aortic bifurcation. The uterine artery is most often the second branch of the anterior division of the internal iliac artery, the first branch being the vesical artery supplying the bladder. The uterine artery can be catheterized with a $4 \mathrm{~F}$ or $5 \mathrm{~F}$ tapered catheter such as a Cobra, MPA or Vertebral catheter or with a microcatheter. The use of microcatheters in combination with reduced guidewire manipulation in the uterine artery is recommended by some (Level 5) as it will usually prevent spasm.

Spasm of the uterine artery is the main cause of technical failure as this will make flow-guided embolization impossible. Spasmolytics are usually not very effective to solve this problem, catheter retrieval and waiting may help (Level 5).

Contrast injection is performed in order to confirm correct positioning of the catheter, the absence of collateral pathways for example to the ovaries and the lack of back flow which can cause non-target embolization of the pelvic organs or the lower extremities. Avoiding embolization of cervical branches is reported to be important to avoid vaginal necrosis. This may be achieved by placing the embolization catheter distal to these branches. In case of blood supply to the fibroids by the ovarian artery, selective microcatheter embolization of the fibroids only can be considered. Some authors recommend the use of PVA particles larger than $500 \mu$ or even microspheres larger than $700 \mu$ in such cases to reduce potential damage to the ovaries [25]. 
When the position of the catheter(s) is satisfactory, UAE can be performed, usually with either PVA particles, PVA microspheres, tris-acryl gelatine (TAGM), Acrylamido PVA microspheres or gelfoam.

Each of the embolic agents has its specific diameter and endpoint for embolization. Thus, the operator should be familiar with the behaviour of the type of embolic agent that is used.

There are several small-sized series in the literature that compare the use of different embolizing agents. However, on comparing embolic agents used in UAE, there is no evidence of superiority of any one embolic agent.

A SR and meta-analysis of published comparative trials comparing embolic agents used in UAE reports no evidence of superiority of any embolic agent. Although there was a trend towards greater uterine and dominant fibroid volume reductions with TAGM compared with spherical PVA, the difference was not statistically significant (Level 2A) [26-33].

\section{Medication and Peri-Procedural Care}

On the day of the procedure, patients should only receive a light meal before embolization because nausea can be encountered after the procedure as a part of the postembolic syndrome.

Premedication with voltarol suppositories (diclofenac) is often used.

In many institutes, a Foley catheter is placed into the bladder before the procedure in order to prevent discomfort during and post procedure.

An iv drip is mandatory in order to administer medication when necessary.

Local analgesics such as Lidocaine $1 \%$ should be administered before puncturing the common femoral artery. US guidance may be used to facilitate puncture of the artery.

Antibiotic prophylaxis, such as cefalozin $1 \mathrm{~g}$, is administered intravenously in many institutes according to gynaecologic protocols. However, there is no proof to support this in any of the controlled trials, and it is therefore not recommended routinely.

For nausea prophylaxis and treatment dolasetron mesylate, metroclopramide or prochlorperazine may be administered.

Many analgesic protocols have been reported for UAE such as mild conscious sedation with (usually up to $50-100 \mu \mathrm{g}$ ) fentanyl and (5 mg of) midazolam, pain-controlled analgesia (PCA) with either fentanyl or morphin, epidural anaesthesia and even general anaesthesia.

It is important to acknowledge that UAE may cause significant post-procedural pain most likely due to ischemic changes within the fibroids and the surrounding myometrium. It is also important to note that pain is usually not experienced during the procedure but may begin in the immediate post-UAE period and is most intense during the first $24 \mathrm{~h}$ after UAE with a peak around $7 \mathrm{~h}$ [32, 3437].

Specific local pain management protocols should be assessed in close cooperation with the departments of anaesthesiology and gynaecology. Patients should have access to additional analgesics as required such as oral paracetamol.

With the combination of appropriate patient instruction, informed consent, intravenous PCA and anti-inflammatory drugs, most patients have satisfactory pain control during the post-UAE period. In rare cases of uncontrollable pain, epidural anaesthesia may be used.

Post-procedural Follow-up and Care

Most complications after UAE are seen in the first week after discharge. As mentioned post-embolic syndrome is not a complication and can be treated with NSIAD's. The rare fatal complications reported have mostly been related to a combination of infection and lack of communication between the patient and treating physician leading to fatal septic shock. [38] 24/7 access to emergency gynaecological care after UAE is crucial. A follow-up telephone call after one week is standard in some institutions. The maximal/ final effect of UAE is seen at 6 months, which is therefore the best timing for clinical follow-up. Patients should know that within the first months, the initial complaints might seem unchanged. Return to normal activities and work is almost always possible 4-6 weeks after UAE.

\section{Outcome and Effectiveness}

Compared with hysterectomy, UAE results in lower blood loss, shorter hospital stay, and quicker resumption of work in the short-term, Mid- and long-term results (5 years) show comparable health-related quality-of-life results and a higher reintervention rate in UAE. Patients treated with hysterectomy and UAE are equally satisfied. The expected 5 years prevention of hysterectomy after successful UAE is $75 \%-80 \%$ following the randomized trials. [39] In singlearm, non-randomized studies and registries, the results for UAE are far better, with a secondary hysterectomy of less than $10 \%$. [40, 41] This is partly due to patient selection, being more "real-life", and the always restricted in- and exclusion criteria of a RCT.

The randomized trials looked specific at heavy menstrual blood loss as inclusion criteria for these trials, and other fibroid-related complaints were not a primary endpoint. Thus, for pain and bulk-related symptoms, it is 
difficult to make any evidence-based conclusion about the efficacy of UAE for these symptoms.

Both UAE and myomectomy result in significant and equal improvements in quality of life. UAE is associated with a shorter hospital stay and fewer major complications but a higher rate of reintervention (Level 1B) [42, 43].

The effect of UAE on fertility has not been well investigated. Conflicting results regarding the effect of UAE on pregnancy and miscarriage rates have been reported [42, 44-46]. Unfortunately, the largest prospective randomized trial that compared UAE with myomectomy, did not report on fertility because ethical approval had been denied to study this outcome [42]. Thus, at present, UAE in women with a child wish should probably be reserved for women in whom myomectomy is not feasible according to gynaecological counselling or for research settings (Level 2A).

\section{Complications}

Major complications of both hysterectomy and UAE are rare and equal in the randomized trials. [47]. Major complications are reported in up to $5 \%$ during and within the first month of the procedure [40, 41, 47]. Pulmonary embolism and deep vein thrombosis are reported in less than $1 \%$. Transcervical expulsion of fibroid tissue, occasionally necessitating surgery in $\pm 5 \%$ of procedures and major infection in $2.5 \%$. There have been three reported deaths due to UAE. In randomized clinical trials, common complications are discharge and fever $(4 \%)$, technical failure (4\%) and post-embolization syndrome (3\%) [39]. Amenorrhea is reported in $\pm 4 \%$ of women, permanent in less than $2 \%$.

\section{Conclusions}

- UAE is a true alternative to hysterectomy in women who want to preserve their uterus (level 1).

- The 5-year outcome in QOL is equal in both groups with no difference in major complications (level 1).

- A 15-20\% hysterectomy rate has to be expected during follow-up after successful embolization (level $1)$.

- In the short-term, UAE had lower blood loss, shorter hospital stay and quicker resumption of work (level 1).

- The risk for ovarian dysfunction after UAE seems overestimated in women $<40$ years of age.

- Every symptomatic patient with uterine myoma's should be offered UAE as alternative treatment to hysterectomy/myomectomy.
- UAE should be incorporated in the (national) gynaecological guidelines.

Acknowledgments $H$. van Overhagen has received grants and has grants pending from COOK, Boston, Abbott, Cordis and Angiodynamics. In addition, he has received payment for lectures, travel and accommodations reimbursement from these companies. Grants and payments are not related to the present manuscript.

Conflict of interest J. A. Reekers has no conflict of interest.

\section{References}

1. Ravina et al (1995) Arterial embolization to treat uterine myomata. Lancet 346:671-672

2. The Rest investigators (2007) Uterine-artery embolization versus surgery for symptomatic uterine fibroids. $N$ Engl $J$ Med 356:360-370

3. Hehenkamp WJK, Volkers NA, Birnie E, Reekers JA, Ankum WM (2008) Symptomatic uterine fibroids: treatment with uterine artery embolization or hysterectomy. Results from the randomized clinical embolization versus hysterectomy (EMMY) trial. Radiology 246:823-832

4. Spielmann AL, Keogh C, Forster BB, Martin ML, Machan LS (2006) Comparison of MRI and sonography in the preliminary evaluation for fibroid embolization. Am J Roentgenol 187:1499-1504

5. Dueholm M, Lundorf E, Hansen ES, Ledertoug S, Olesen F (2002) Accuracy of magnetic resonance imaging and transvaginal ultrasonography in the diagnosis, mapping and measurement of uterine myomas. Am J Obstet Gynecol 186:409-415

6. Volkers NA, Hehenkamp WIK, Spijkerboer AM, Moolhuijzen AD, Birnie E, Ankum WM, Reekers JA (2008) MR reproducibility in the assessment of uterine fibroids for patients scheduled for uterine artery embolization. Cardiovasc Interv Radiol 31:260-268

7. Kirby JM, Burrows D, Haider E, Maizlin M, Midia M (2011) Utility of MRI before and after uterine artery embolization: why to do it and what to look for. Cardiovasc Interv Radiol 34:705-716

8. Williams PL, Coote JM, Watkinson AF (2011) Pre-uterine artery embolization MRI: beyond fibroids. Cardiovasc Interv Radiol 34:1143-1150

9. McLucas B, Goodwin S, Adler L, Rappaport A, Reed P (2001) Pregnancy following uterine fibroid embolization. Int J Gynaecol Obstet 74:1-7

10. Ravina JH, Vigneron NC, Aymard A, Le Dref O, Merland JJ (2000) Pregnanct after embolization of uterine myoma: report of 12 cases. Fertil Steril 73:1241-1243

11. Parthipun AA, Taylor J, Manyonda I, Belli AM (2010) Does size really matter? Analysis of the effect of large fibroids and uterine volumes on complication rates of uterine artery embolization. Cardiovasc Interv Radiol 33:955

12. Smeets AJ, Nijenhuis RJ, van Rooij WJ et al (2010) Uterine artery embolization in patients with a large fibroid burden: longterm clinical and MR follow-up. Cardiovasc Interv Radiol 33:943-948

13. Goodwin SC, Bonilla SC, Sacks D et al (2003) Reporting standards for uterine artery embolization for the treatment of uterine leiomyomata. J Vasc Interv Radiol 14(suppl):S457-S476

14. Margau R, Simons ME, Rajan DK et al (2008) Outcomes after uterine artery embolization for pedunculated subserosal leiomyomas. J Vasc Interv Radiol 19:657-661 
15. Smeets AJ, Nijenhuis RJ, Boekkooi PF, Vervest HA, van Rooij WJ, Lohle PN (2010) Is an intrauterine device a contraindication for uterine artery embolization? A study of 20 patients. J Vasc Interv Radiol 21:272-274

16. Andrews TA, Spies JB, Sacks D et al (2004) Patient care and uterine artery embolization for leiomyomata. J Vasc Interv Radiol $15: 115-120$

17. Stacul F, van der Molen AJ, Reimer P et al (2011) Contrast induced nephropathy: updated ESUR Contrast Media Committee Guidelines. Eur Radiol 21(12):2527-2541

18. Kim MD, Lee M, Lee MS, Park SI, Wong JY, Lee do Y, Lee KH (2012) Uterine artery embolization of large fibroids: comparative study of procedure with and without pretreatment gonadotropin releasing hormone agonists. Am J Roentgenol 199:441-446

19. Vetter S, Schulz FW, Strecker EP, Zoetelief J (2004) Patient exposure in uterine artery embolization of leiomyomata: calculation of organ doses and effective doses. Eur Radiol 14:842-848

20. Nikolic B, Spies JB, Lundsten MJ, Abbara S (2000) Patient radiation dose associated with uterine artery embolization. Radiology 214:121-125

21. Glomset O, Hellesnes J, Heimland N, Hafsahl G, Smith HJ (2006) Assessment of organ radiation dose associated with uterine artery embolization. Acta Radiol 2:179-185

22. Sapoval M, Pellerin O, Rehel JL et al (2010) Uterine artery embolization for leiomyomata: optimization of the radiation dose to the patient using a flat-panel detector angiographic suite. Cardiovasc Interv Radiol 33:949-954

23. Nikolic B, Abbara S, Levy E et al (2000) Influence of radiographic technique and equipment on the absorded ovarian dose associated with uterine artery embolization. J Vasc Interv Radiol 11:1173-1178

24. Costantino M, Lee J, Mc Cullough M, Nsrouli-Maktabi H, Spies JB (2010) Bilateral versus unilateral femoral access for uterine artery embolization: results of a randomized comparative trial. J Vasc Interv Radiol 21:829-835

25. Scheurig-Muenkler C, Poellinger A, Wagner M, Hamm B, Kroencke TJ (2011) Ovarian artery embolization in patients with collateral supply to symptomatic uterine leiomyomata. Cardiovasc Interv Radiol 34:1199-1207

26. Bilhim T, Pisco J, Duarte M, Oliveira AG (2011) Polyvinyl alcohol particle size for uterine artery embolization: a prospective randomized study of initial use of 350-500 $\mu \mathrm{m}$ particles versus initial use of 500-700 $\mu \mathrm{m}$ particles. J Vasc Interv Radiol 22:21-27

27. Rasuli P, Hammond I, Al-Mutairi B et al (2008) Spherical versus conventional polyvinyl alcohol particles for uterine artery embolization. J Vasc Interv Radiol 19:42-46

28. Spies JB, Allison S, Flick P et al (2005) Spherical polyvinyl alcohol versus tris-acryl gelatine microspheres for uterine artery embolization for leiomyomas: results of a limited randomized comparative study. J Vasc Interv Radiol 16:1431-1437

29. Siskin GP, Schuster M, Mandato K, Englander M, Herr A (2008) Leiomyoma infarction after uterine artery embolization: a prospective randomized study comparing tris-acryl gelatin microspheres versus polyvinyl alcohol microspheres. J Vasc Interv Radiol 19:58-65

30. Yu SCH, Lok I, Ho SSY, Tong MMB, Hui JWY (2011) Comparison of clinical outcomes of tris-acryl microspheres versus polyvinyl alcohol microspheres for uterine artery embolization for leiomyomas: results of a randomized trial. J Vasc Interv Radiol 22:1229-1235

31. Worthington-Kirsch RL, Siskin GP, Hegener P, Chesnick R (2011) Comparison of the efficacy of the embolic agents acrylamido polynivyl alcohol microspheres and tris-acryl gelatine microspheres for uterine artery embolization for leiomyomas: a prospective randomized controlled trial. Cardiovasc Interv Radiol 34:493-501

32. Sone M, Arai Y, Shimizu T, Takeuchi Y, Higashihara H, Ohgi S, Ishiguchi T (2010) Phase I/II multiinstitutional study of uterine artery embolization with gelatine sponge for symptomatic uterine leiomyomata: Japan Interventional Radiology in Oncology Study Group Study. J Vasc Interv Radiol 21:1665-1671

33. Das R, Champaneria R, Daniels JP, Belli AM (2013) Comparison of embolic agents used in uterine artery embolisation: a systematic review and meta-analysis. Cardiovasc Interv Radiol. doi:10.1007/s00270-013-0790-0

34. Roth AR, Spies JB, Walsh SM, Wood BJ, Gomez-Jorge J, Levy EB (2000) Pain after uterine artery embolization for leiomyomata: can its severity be predicted and does severity predict outcome? J Vasc Interv Radiol 11:1047

35. Pron G, Mocarski E, Bennett J et al (2003) Tolerance, hospital stay and recovery after uterine artery embolization for fibroids: the Ontario uterine fibroid embolization trial. J Vasc Interv Radiol 14:1243-1250

36. Hehenkamp W, Volkers NA, Birnie E, Reekers JA, Ankum WM (2006) Pain and return to daily activities after uterine artery embolization and hysterectomy in the treatment of symptomatic uterine fibroids: results from the randomized EMMY trial. Cardiovasc Interv Radiol 29:179-187

37. Kim HS, Czuczman GJ, Nicholson WK, Pham LD, Richman JM (2008) Pain levels within 24 hours after UAE: a comparison of morphine and fentanyl patient-controlled analgesia. Cardiovasc Interv Radiol 31:1100-1107

38. De Blok S, de Vries C, Prinssen HM, Blauwgeers HL, JornaMeijer LB (2003) Fatal sepsis after uterine artery embolization with microsheres. J Vasc Interv Radiol 14:779-783

39. Van der Kooij SM, Bipat S, Hehenkamp WJ, Ankum WM, Reekers JA (2011) Uterine artery embolization versus surgery in the treatment of symptomatic fibroids: a systematic review and metaanalysis. Am J Obstet Gynecol 205(105):e1-e13

40. Goodwin SC, Spies JB (2009) Uterine fibroid embolization. N Engl J Med 361:690-697

41. Bulman JC, Ascher SM, Spies JB (2012) Current concepts in uterine fibroid embolization. Radiographics 32:1735-1750

42. Manyonda IT, Bratby M, Horst JS, Banu N, Gorti M, Belli AM (2012) Uterine artery embolization versus myomectomy: impact on quality of life-results of the fume (fibroids of the uterus: myomectomy versus embolization) trial. Cardiovasc Interv Radiol 35:530-536

43. Mara M, Maskova J, Fucikova Z, Kuzel D, Belsan T, Sosna O (2008) Midterm clinical and first reproductive results of a randomized controlled trial comparing uterine fibroid embolization and myomectomy. Cardiovasc Interv Radiol 31:73-85

44. Firouznia K, Ghanaati H, Sanaati M, Jalali AH, Shakiba M (2009) Pregnancy after uterine artery embolization for symptomatic fibroids: a series of 15 pregnancies. AJR 192:1588-1592

45. Homer H, Saridogan E (2010) Uterine artery embolization for fibroids is associated with an increased risk of miscarriage. Fertil Steril 94:324-330

46. Gupta JK, Sinha A, Lumsden MA, Hickey M (2012) Uterine artery embolization for symptomatic uterine fibroids. Cochrane Database Syst Rev 5:CD005073

47. Martin J, Bhanot K, Athreya S (2013) Complications and reinterventions in uterine artery embolization for symptomatic uterine fibroids: a literature review and meta analysis. Cardiovasc Interv Radiol 36:395-402 